\title{
The role of biological nitrogen fixation by non-legumes in the sustainable production of food and biofuels
}

\author{
Euan K. James • J. Ivo Baldani
}

Received: 28 May 2012 / Accepted: 31 May 2012 /Published online: 9 June 2012

(C) Springer Science+Business Media B.V. 2012

The 12th International Symposium on biological nitrogen fixation (BNF) with Non-Legumes took place in Buzios, Rio de Janeiro, Brazil from October 3 to 8, 2010. It was the latest in a series of meetings that, fittingly, first began in Brazil, in 1977 in the city of Piracicaba. This Special Issue of Plant and Soil comprises 21 papers from the Buzios Symposium, as well as a commentary and three obituaries. Given the volatility (and general upward trend) in oil prices and global attempts to alleviate greenhouse gas (GHG) emissions associated with the agricultural use of $\mathrm{N}$ containing mineral fertilizers produced by the energyintensive Haber-Bosch process, it was inevitable that the main focus of the 2010 Symposium would be very much on the substitution of fertilizers in favour of the increased use of BNF in non-legume cropping systems. In the intervening years since the last time the symposium was held in Brazil (August 1987 in Rio de Janeiro), knowledge about non-legume BNF has increased enormously, encompassing everything from

Responsible Editor: Hans Lambers.

E. K. James $(\bowtie)$

The James Hutton Institute, Invergowrie, Dundee DD2 5DA, UK

e-mail: euan.james@hutton.ac.uk

J. I. Baldani

Embrapa Agrobiologia,

BR 465, km07,

Seropédica, Rio de Janeiro 23890-000, Brazil more accurate ${ }^{15} \mathrm{~N}$-based quantification studies in the field through to the molecular identification of their associated diazotrophs (both culturable and unculturable) and the localization of these bacteria via high resolution microscopy. The completely sequenced genomes of many diazotrophic bacteria are also now available, and more are deposited in databases every year. Indeed, such technologies, which only a few years ago were considered remarkable, are now becoming standard tools in this field.

In the case of sugarcane and other biofuel crops, the location of the 2010 meeting in Brazil was especially pertinent, as the highly advanced Brazilian bioethanol programme, which produces over 27 billion litres of ethanol per year, is based upon the cultivation of sugarcane, and Brazilian cane has long been known to be able to derive much of its N-requirements via BNF. The consequent low fertilizer use by Brazilian cane producers not only makes for enormous economic savings, but also mitigates against GHG emissions from the production of N-containing fertilizers, as well as their transport and application and nitrous oxide emissions. However, in spite of the strong likelihood that cane can benefit significantly from BNF, little is known about how much $\mathrm{N}$ it can really fix, and as field-based BNF quantification studies may take several years to complete, we are thus fortunate to include two such studies in this Special Issue (both utilizing the ${ }^{15} \mathrm{~N}$ natural abundance technique): one on sugarcane by Urquiaga et al., and one on the C-4 "energy crop" 
elephant grass (Pennisetum purpureum) by de Morais et al. Both studies demonstrate very substantial inputs into these crops via BNF of at least $40 \mathrm{~kg} \mathrm{~N} \mathrm{ha}^{-1} \mathrm{yr}^{-1}$ for sugarcane and up to $132 \mathrm{~kg} \mathrm{~N} \mathrm{ha}^{-1} \mathrm{yr}^{-1}$ for elephant grass. Interestingly, another ${ }^{15} \mathrm{~N}$-based quantification study, but this time a greenhouse-based one from Uruguay by Taule et al., has demonstrated potentially very substantial inputs by BNF in local cultivars, thus suggesting that sugarcane BNF is not just a Brazilian phenomenon. On the bacterial side, the specific diazotrophic organisms responsible for BNF in sugarcane have yet to be identified, but more and more diverse diazotrophic organisms are being isolated both from the rhizosphere and surfaces of these plants, as well as from within them ("endophytic diazotrophs"). Three studies of culturable diazotrophs isolated from energy crops are included in this issue: Videira et al. on elephant grass in Brazil, Grönemeyer et al. on elephant grass, sorghum (Sorghum bicolor) and maize (Zea mays) in Namibia, and another by Taule et al. on sugarcane in Uruguay. In addition, non-culturable techniques based upon bacterial gene transcripts, such as those from the $16 \mathrm{~S}$ rRNA and nifH genes, are being increasingly utilised to detect and identify a much wider and more diverse range of diazotrophs in field-grown material (see Fischer et al.; Vollú et al. in this issue).

The role of BNF and/or plant growth-promoting rhizobacteria (PGPR) in the production of nonlegume food crops is also featured in this Special Issue. Many researchers have continued to focus on rice (Brusamarello-Santos et al., Vargas et al.), as it is so important worldwide as a staple food, and increased future demand will clearly entail an increased application of fertilizer N. Indeed, it is estimated that twice as much fixed $\mathrm{N}$ will be required to raise rice production by 2020 to meet the food requirements of the increasing human population, but as with biofuel crops, an alternative strategy is to utilize BNF and PGPR more widely in rice cultivation. To this end, Azospirillum, which has long been known to be an effective diazotrophic PGPR on rice and other cereals, is strongly featured in this special issue. The papers by CastroGuerrero et al., Walker et al. and Vargas et al. have examined in detail at the biochemical and/or molecular level the mechanisms by which cereals such as rice, maize and wheat (Triticum aestivum) respond to inoculation by Azospirillum strains (and also how the bacteria respond to their hosts). Similarly, Brusamarello-Santos et al. have examined the molecular response of rice to inoculation with another diazotroph, Herbaspirillum seropedicae. Interestingly, the growth-promoting properties of PGPRs, such as Azospirillum are not confined to grasses/cereals, as Star et al. in this issue have examined the ability of Azospirillum strains to release auxins that can promote the ability of a legume (Vicia sativa) to be nodulated by Rhizobium.

Work has continued on "model" diazotrophs/PGPRs for BNF and growth promotion in grasses (Poaceae) and other non-legumes, and the most prominent of these have now been sequenced (Azoarcus, Azospirillum spp., Burkholderia spp., Gluconacetobacter diazotrophicus and H. seropedicae). Although these culturable diazotrophs are by no means claimed to be "representative" of the enormous diversity of bacteria that are likely to be associated with non-legumes (both culturable and nonculturable), they have provided an insight into how non-legumes, particularly Poaceae, can interact intimately with associated beneficial diazotrophs in a non-symbiotic (i.e. non-nodulating) manner. In addition to being models, many of these diazotrophs are now being developed as inoculants (often using mixtures of species/strains), and one of the most promising of the "endophytic diazotrophic" bacterial genera with respect to being both a model and a prospective inoculant is Herbaspirillum. Two major reviews on this genus are featured in this Special Issue; Chubatsu et al., who explain how $H$. seropedicae regulates $\mathrm{N}$ fixation, and Monteiro et al. who review for the first time the (generally) beneficial interactions between the members of this fascinating and diverse genus and their various Poaceous hosts. Continuing with this theme of how model diazotrophs interact with specific Poaceous hosts under controlled conditions, Alquéres et al. have demonstrated how a very different kind of model diazotroph, the acid- and sugar-loving species G. diazotrophicus, may regulate its $\mathrm{N}$-fixation within its hosts (particularly sugarcane) via its ability to control intracellular asparagine biosynthesis. Finally, on a nonPoaceous note, Weyens et al. show that the fast-growing biofuel tree, poplar (Populus deltoides), can have its growth greatly promoted by the (non-diazotrophic) PGPR strain Pseudomonas putida W619, and that this endophytic bacterium can inhabit the xylem vessels in a non-pathogenic manner. Interestingly, however, Weyens et al. also have a note of caution in their study, as the gfp-variant strains they used for their localization studies were greatly impaired in colonization and actually had a negative effect on poplar growth. 
Where do we go from here? It could be argued that as the concept of sugarcane (and rice) BNF is now sufficiently well established and quantified (at least in a South American context), and that the amount of $\mathrm{N}$ fixed depends upon the plant genotype and growing conditions, that it is now ready to be more fully exploited (i.e. optimized) by plant breeders working closely with agronomists (and possibly plant physiologists to try and identify the mechanisms by which fixed $\mathrm{N}$ is assimilated and processed by those varieties that can utilize it). Microbiologists will also, inevitably, still play a major role in the future utilization of BNF in sugarcane, but most likely in an increasingly more applied manner e.g. da Silva et al. in this issue have started to develop technologies specifically aimed at providing sugarcane farmers in Brazil with an effective inoculant consisting of several diazotrophs. Indeed, these workers (and others before them) have demonstrated that although the exact nature of the diazotrophs that fix $\mathrm{N}$ and/or promote growth in cereals and sugarcane may only be deciphered many years from now, they are most likely to belong to consortia of bacteria both on and within the plant. The members of these consortia are likely to shift with plant genotype, growing conditions, growth stage, soil and environment, and it is significant that each new study of diazotrophs in non-legumes, whether they utilize "traditional" culturing techniques or non-culturable molecular methods, always result in discoveries of novel diazotrophs, as exemplified by several papers in this issue (Ambrosini et al.; Fischer et al.; Grönemeyer et al.; Jha et al.; Taule et al., Videira et al.; Vollú et al.). "Matching" sugarcane and rice genotypes to these shifting consortia in order to optimize BNF will be an enormous challenge to breeders, agronomists, soil scientist and microbiologists, but if it can be achieved it will reap rich rewards.

With regard to PGPRs, the development of biofertilizers that operate through multiple mechanisms, and not just through BNF is also continuing apace and are represented in this issue e.g. Walker et al. have been examining a highly effective mixed inoculant containing diazotrophs, PGPR and mycorrhiza for maize in France, and Tortora et al. have been investigating another aspect of Azospirillum plant growth promotion i.e. its potential as an agent of biological control against fungal pathogens.

Finally, in this Special Issue we celebrate the lives and scientific careers of three recently deceased scientists who contributed so much by pioneering research into BNF by either (or both) non-legumes and legumes: Bob Burris, Jesús Caballero-Mellado and John Witty. We also celebrate the 125th anniversary of MV. Galippe, who first introduced the concept of the endophytic microbe.

We acknowledge the tremendous work of the expert referees who reviewed the papers in this Special Issue in a timely and professional manner, and we would also like to thank those people who assisted Prof. Ivo Baldani in organizing the Symposium from which these papers were taken i.e. R.M. Boddey, A. Hemerley, F.L. Olivares, V.M. Reis, and E.M. Souza. We also acknowledge financial support from CNPq, CAPES, FAPERJ, FUNDAÇÃO AGRISUS and EMBRAPA which allowed us to present the Symposium and to bring to it so much international expertise in the field of nonlegume BNF. Special thanks are due to the National Institute for Science and Technology on Biological Nitrogen Fixation ( INCT-FBN) which supported many of the students attending from various Brazilian Institutions. 